\title{
On the P-formulation and the Split-Fraction-Formulation for the Generalized Pooling Problem
}

\author{
Xin Cheng ${ }^{1}$ and Xiang $\mathrm{Li}^{1}$ \\ ${ }^{1}$ Queen's University
}

July 21, 2021

\begin{abstract}
The generalized pooling problem (GPP) is a NP-hard problem for which the solution time for securing a global optimal solution heavily depends on the strength of the problem formulation. The existing GPP formulations use either quality variables (Pformulation and the variants) or split-fraction variables (SF-formulation and the variants) to model the material balance at the pools. This paper is the first attempt to develop theoretical results for comparing the strength of P-formulation and $\mathrm{SF}$-formulation. It is found that, an enhanced version of $\mathrm{P}$-formulation, called $\mathrm{P}^{+}$-formulation, is at least as strong as $\mathrm{SF}$ formulation under mild conditions. Furthermore, $\mathrm{P}^{+}$-formulation becomes identical to $\mathrm{P}$-formulation when the pooling network comprises only mixers and splitters. With additional conditions that are often satisfied at the root node, P-formulation is proved to be as least as strong as SF-formulation. The theoretical results are verified by the computational study of 23 problem instances.
\end{abstract}

\section{Hosted file}

Cheng\&Li2021.pdf available at https://authorea.com/users/426886/articles/531235-on-the-pformulation-and-the-split-fraction-formulation-for-the-generalized-pooling-problem 